\title{
A stochastic equivalence approach for an Ornstein-Uhlenbeck process driven power system dynamics
}

\author{
R. H. HIRPARA
}

\begin{abstract}
This paper develops a stochastic equivalence approach for an Ornstein-Uhlenbeck process-driven power system. The concept of stochastic equivalence coupled with stochastic differential rule plays the important role to develop the stochastic equivalence approach of this paper. This paper also develops the prediction theory of power system dynamics with the OU process as well.
\end{abstract}

Mathematics Subject Classification 2000: 60H10, 93E03.

Additional Key Words and Phrases: stochastic equivalence approach, stochastic differential equation, the OU process, Kuramoto oscillator, power system dynamics.

\section{INTRODUCTION}

The stochastic differential equation (SDE) is caused by random term. This random term can be represented in form of white noise process or coloured noise process. For deterministic representation of the swing equation of a single machineinfinite bus (SMIB) system, see Kundur (1994). The white noise driven-Itô SDE model for a power system dynamics, see (Wang and Crow 2013; Hirpara and Sharma 2015). The white noise driven-Stratonovich SDE model for a power system dynamics and circuits and systems, see (Hirpara 2019; Patil and Sharma 2015) and the coloured noise driven-SDE model for a power system dynamics, see(Hänggi and Jung 1995; Patel and Sharma 2012; Patil and Sharma 2014; Hirpara and Sharma 2015; Guo and Shi 2017) and recent publication Verdejo et al. (2019) as well. The well-known Kuramoto model (Dörfler and Bullo 2010; Tönjes 2010; Simpson-Porco 2012; Schäfer et al. 2017; Supplemental material 2017), explaining essential mechanisms underlying synchronization, is very similar to the power system dynamics when neglecting inertia. 
The problem becomes complex when the extended phase space approach increases the dimensionality of the augmented state vector with the Markovian property, see Patel and Sharma (2012). It becomes easier approaches that allow the Markovian property as well as reduce the dimensionality of the state vector and that is known as the stochastic equivalence approach, see Patil and Sharma (2014) for greater detail.

This paper is aimed to analyse power system dynamics with the stochastic equivalence approach. In contrast to Hirpara and Sharma (2015), we developed stochastic power system dynamics in the presence of an Ornstein-Uhlenbeck (OU) process with the stochastic equivalence approach and examine the effectiveness of the prediction equations with no observations or valueless observations. The Numerical simulations help to check stochastic equivalent SDE in MATLAB for a smaller correlation time.

The remainder of the paper is organized as follows. Section 2 describes mathematical preliminaries. Section 3 is about the stochastic equivalence approach for an OU driven power system dynamics and numerical simulations. Section 4 discusses concluding remarks.

\section{MATHEMATICAL PRELIMINARIES}

Consider the stochastic differential system described by

$$
\dot{\mu}_{t}=A\left(\mu_{t}\right)+B\left(\mu_{t}\right) \eta_{t}
$$

where the input process $\eta_{t}$ is a stationary process with zero mean, relatively smaller correlation time. The right-hand side $B\left(\mu_{t}\right) \eta_{t}$ of equation (1) has a multiplicative noise character. Two different SDEs can be regarded as the stochastically equivalent, if they are associated with the same Fokker-Planck equation. Importantly, the concept of stochastic equivalence is useful for the coloured noisedriven SDE, where the input noise process is a coloured noise process with a smaller correlation time. A simple calculation shows that the white noise-driven stochastic differential equation associated with the Fokker-Planck equation (Stratonovich 1963, p. 97) assumes the structure 


$$
\dot{\mu}_{t}=\left(S_{1}\left(\mu_{t}\right)-\frac{1}{2} \partial_{\mu_{t}} S_{2}\left(\mu_{t}\right)\right)+\sqrt{S_{2}\left(\mu_{t}\right)} w_{0}(t)
$$

where the input process $w_{0}(t)$ is zero mean, stationary, Gaussian white noise process. Thanks to equation (4.180) of Stratonovich (1963), a special case of equation (4.180), i.e. the coefficients $S_{1}\left(\mu_{t}\right)$ and $S_{2}\left(\mu_{t}\right)$ for the stochastic differential system $\dot{\mu}_{t}=A\left(\mu_{t}\right)+B\left(\mu_{t}\right) \eta_{t}$, can be re-cast as

$$
\begin{gathered}
S_{1}\left(\mu_{t}\right)=A\left(\mu_{t}\right)+\frac{\gamma_{1}}{2} B\left(\mu_{t}\right) \partial_{\mu_{t}} B\left(\mu_{t}\right)+\gamma_{2} B^{2}\left(\mu_{t}\right) \partial_{\mu_{t}} B\left(\mu_{t}\right) \partial_{\mu_{t}}\left(\frac{A\left(\mu_{t}\right)}{B\left(\mu_{t}\right)}\right), \\
S_{2}\left(\mu_{t}\right)=\gamma_{1} B^{2}\left(\mu_{t}\right)+2 \gamma_{2} B^{3}\left(\mu_{t}\right) \partial_{\mu_{t}}\left(\frac{A\left(\mu_{t}\right)}{B\left(\mu_{t}\right)}\right),
\end{gathered}
$$

Where, $\quad \gamma_{1}=2 \int_{-\infty}^{0} R_{\eta \eta}(\tau) d \tau, \quad \gamma_{2}=\int_{-\infty}^{0}|\tau| R_{\eta \eta}(\tau) d \tau, \quad$ and $\quad$ the autocorrelation $R_{\eta \eta}(\tau)=E \eta_{t+\tau} \eta_{t}$. Note that $\partial_{\mu_{t}}=\frac{\partial}{\partial \mu_{t}}$ and $\partial_{\mu_{t}}^{2}=\frac{\partial^{2}}{\partial \mu_{t}^{2}}$ are throughout the paper.

Equations (3)-(4) are valid for the weakly coloured noise input process $\eta_{t}$. Equation (2) in conjunction with equations (3)-(4) assumes the structure

$$
\begin{aligned}
\dot{\mu}_{t}= & A\left(\mu_{t}\right)-\frac{\gamma_{2}}{2} B^{2}\left(\mu_{t}\right) \partial_{\mu_{t}} B\left(\mu_{t}\right) \partial_{\mu_{t}}\left(\frac{A\left(\mu_{t}\right)}{B\left(\mu_{t}\right)}\right)-\frac{\gamma_{2}}{2} B^{3}\left(\mu_{t}\right) \partial_{\mu_{t}}^{2}\left(\frac{A\left(\mu_{t}\right)}{B\left(\mu_{t}\right)}\right) \\
& +\sqrt{\gamma_{1}} B\left(\mu_{t}\right) \sqrt{1+\frac{2 \gamma_{2}}{\gamma_{1}} B\left(\mu_{t}\right) \partial_{\mu_{t}}\left(\frac{A\left(\mu_{t}\right)}{B\left(\mu_{t}\right)}\right)} w_{0}(t),
\end{aligned}
$$

Note that equation (5) is stochastically equivalent to the SDE, i.e. $\dot{\mu}_{t}=A\left(\mu_{t}\right)+B\left(\mu_{t}\right) \eta_{t}$, since both are associated with the same Fokker-Planck equation. Equation (5) can be're-formulated' in the Itô sense, i.e

$$
\begin{aligned}
d \mu_{t}=( & \left.A\left(\mu_{t}\right)-\frac{\gamma_{2}}{2} B^{2}\left(\mu_{t}\right) \partial_{\mu_{t}} B\left(\mu_{t}\right) \partial_{\mu_{t}}\left(\frac{A\left(\mu_{t}\right)}{B\left(\mu_{t}\right)}\right)-\frac{\gamma_{2}}{2} B^{3}\left(\mu_{t}\right) \partial_{\mu_{t}}^{2}\left(\frac{A\left(\mu_{t}\right)}{B\left(\mu_{t}\right)}\right)\right) d t \\
& +\sqrt{\gamma_{1}} B\left(\mu_{t}\right) \sqrt{1+\frac{2 \gamma_{2}}{\gamma_{1}} B\left(\mu_{t}\right) \partial_{\mu_{t}}\left(\frac{A\left(\mu_{t}\right)}{B\left(\mu_{t}\right)}\right)} d W_{t}
\end{aligned}
$$

where 


$$
\begin{aligned}
& a\left(\mu_{t}\right)=A\left(\mu_{t}\right)-\frac{\gamma_{2}}{2} B^{2}\left(\mu_{t}\right) \partial_{\mu_{t}} B\left(\mu_{t}\right) \partial_{\mu_{t}}\left(\frac{A\left(\mu_{t}\right)}{B\left(\mu_{t}\right)}\right)-\frac{\gamma_{2}}{2} B^{3}\left(x_{t}\right) \partial_{x_{t}}^{2}\left(\frac{A\left(x_{t}\right)}{B\left(x_{t}\right)}\right), \\
& b\left(\mu_{t}\right)=\sqrt{\gamma_{1}} B\left(\mu_{t}\right) \sqrt{1+\frac{2 \gamma_{2}}{\gamma_{1}} B\left(\mu_{t}\right) \partial_{x_{t}}\left(\frac{A\left(\mu_{t}\right)}{B\left(\mu_{t}\right)}\right)} .
\end{aligned}
$$

Equation (6), the Itô stochastic differential equation, describes a rigorous interpretation of the SDE in contrast to equation (5).

The OU process is a Gauss-Markov process and satisfies the stochastic differential equation,

$$
d \eta_{t}=-\frac{1}{\tau_{c o r}} \eta_{t} d t+\frac{\sqrt{2 N}}{\tau_{c o r}} d W_{t}
$$

where the terms $\tau_{c o r}$ and $\frac{\sqrt{2 N}}{\tau_{c o r}}$ has an interpretation as correlation time and the process noise coefficient. Furthermore, the autocorrelation $R_{\eta \eta}(\tau)$ of the $\mathrm{OU}$ process, a stationary process, is $\frac{N}{\tau_{c o r}} e^{-\frac{|\tau|}{\tau_{c o r}}}$. This expression suggests that the correlation time of the stationary coloured noise can be explained using the concept of the autocorrelation of the coloured noise process. For the OU process, a weakly coloured noise process, the terms $\gamma_{1}, \gamma_{2}$ of equations (3)-(4) become,

$$
\gamma_{1}=2 N, \gamma_{2}=N \tau_{c o r} \text {. }
$$

From equation (10) and equations (7)-(8), we have the following system nonlinearity $a\left(\mu_{t}\right)$ and process noise coefficient $b\left(\mu_{t}\right)$ for the OU process-driven stochastic differential system:

$$
\begin{aligned}
& a\left(\mu_{t}\right)=A\left(\mu_{t}\right)-\frac{N \tau_{c o r}}{2} B^{2}\left(\mu_{t}\right) \partial_{\mu_{t}} B\left(\mu_{t}\right) \partial_{\mu_{t}}\left(\frac{A\left(\mu_{t}\right)}{B\left(\mu_{t}\right)}\right)-\frac{N \tau_{c o r}}{2} B^{3}\left(\mu_{t}\right) \partial_{\mu_{t}}^{2}\left(\frac{A\left(\mu_{t}\right)}{B\left(\mu_{t}\right)}\right), \\
& b\left(\mu_{t}\right)=\sqrt{2 N} B\left(\mu_{t}\right) \sqrt{1+\tau_{c o r} B\left(\mu_{t}\right) \partial_{\mu_{t}}\left(\frac{A\left(\mu_{t}\right)}{B\left(\mu_{t}\right)}\right)} .
\end{aligned}
$$

For a coloured noise-driven SDE, the 'scalar' exact mean and variance evolutions, $d\left\langle\mu_{t}\right\rangle$ and $d P_{t}$ are, 


$$
\begin{aligned}
& d\left\langle\mu_{t}\right\rangle=\left(A\left(\left\langle\mu_{t}\right\rangle\right)-\frac{\gamma_{2}}{2} B^{2}\left(\left\langle\mu_{t}\right\rangle\right) \partial_{\left\langle\mu_{t}\right\rangle} B\left(\left\langle\mu_{t}\right\rangle\right) \partial_{\left\langle\mu_{t}\right\rangle}\left(\frac{A\left(\left\langle\mu_{t}\right\rangle\right)}{B\left(\left\langle\mu_{t}\right\rangle\right)}\right)\right. \\
& -\frac{\gamma_{2}}{2} B^{3}\left(\left\langle\mu_{t}\right\rangle\right) \partial_{\left\langle\mu_{t}\right\rangle}^{2}\left(\frac{A\left(\left\langle\mu_{t}\right\rangle\right)}{B\left(\left\langle\mu_{t}\right\rangle\right)}\right)+\frac{1}{2} P_{t}\left(\partial_{\left\langle\mu_{t}\right\rangle}^{2} A\left(\left\langle\mu_{t}\right\rangle\right)-\frac{\gamma_{2}}{2}\left(B^{3}\left(\left\langle\mu_{t}\right\rangle\right) \partial_{\left\langle\mu_{t}\right\rangle}^{4}\left(\frac{A\left(\left\langle\mu_{t}\right\rangle\right)}{B\left(\left\langle\mu_{t}\right\rangle\right)}\right)\right.\right. \\
& +7 B^{2}\left(\left\langle\mu_{t}\right\rangle\right) \partial_{\left\langle\mu_{t}\right\rangle} B\left(\left\langle\mu_{t}\right\rangle\right) \partial_{\left\langle\mu_{t}\right\rangle}^{3}\left(\frac{A\left(\left\langle\mu_{t}\right\rangle\right)}{B\left(\left\langle\mu_{t}\right\rangle\right)}\right) \\
& +5 B^{2}\left(\left\langle\mu_{t}\right\rangle\right) \partial_{\left\langle\mu_{t}\right\rangle}^{2} B\left(\left\langle\mu_{t}\right\rangle\right) \partial_{\left\langle\mu_{t}\right\rangle}^{2}\left(\frac{A\left(\left\langle\mu_{t}\right\rangle\right)}{B\left(\left\langle\mu_{t}\right\rangle\right)}\right)+10 B\left(\left\langle\mu_{t}\right\rangle\right)\left(\partial_{\left\langle\mu_{t}\right\rangle} B\left(\left\langle\mu_{t}\right\rangle\right)\right)^{2} \partial_{\left\langle\mu_{t}\right\rangle}^{2}\left(\frac{A\left(\left\langle\mu_{t}\right\rangle\right)}{B\left(\left\langle\mu_{t}\right\rangle\right)}\right) \\
& +2\left(\partial_{\left\langle\mu_{t}\right\rangle} B\left(\left\langle\mu_{t}\right\rangle\right)\right)^{3} \partial_{\left\langle\mu_{t}\right\rangle}\left(\frac{A\left(\left\langle\mu_{t}\right\rangle\right)}{B\left(\left\langle\mu_{t}\right\rangle\right)}\right)+6 B\left(\left\langle\mu_{t}\right\rangle\right) \\
& \left.\left.\times \partial_{\left\langle\mu_{t}\right\rangle} B\left(\left\langle\mu_{t}\right\rangle\right) \partial_{\left\langle\mu_{t}\right\rangle}^{2} B\left(\left\langle\mu_{t}\right\rangle\right) \partial_{\left\langle\mu_{t}\right\rangle}\left(\frac{A\left(\left\langle\mu_{t}\right\rangle\right)}{B\left(\left\langle\mu_{t}\right\rangle\right)}\right)\right)\right) d t \\
& d P_{t}=\left(2 P _ { t } \left(\partial_{\left\langle\mu_{t}\right\rangle} A\left(\left\langle\mu_{t}\right\rangle\right)-\frac{\gamma_{2}}{2}\left(4 B^{2}\left(\left\langle\mu_{t}\right\rangle\right) \partial_{\left\langle\mu_{t}\right\rangle} B\left(\left\langle\mu_{t}\right\rangle\right) \partial_{\left\langle\mu_{t}\right\rangle}^{2}\left(\frac{A\left(\left\langle\mu_{t}\right\rangle\right)}{B\left(\left\langle\mu_{t}\right\rangle\right)}\right)\right.\right.\right. \\
& +B^{2}\left(\left\langle\mu_{t}\right\rangle\right) \partial_{\left\langle\mu_{t}\right\rangle}^{2} B\left(\left\langle\mu_{t}\right\rangle\right) \partial_{\left\langle\mu_{t}\right\rangle}\left(\frac{A\left(\left\langle\mu_{t}\right\rangle\right)}{B\left(\left\langle\mu_{t}\right\rangle\right)}\right) \\
& \left.+2 B\left(\left\langle\mu_{t}\right\rangle\right)\left(\partial_{\left\langle\mu_{t}\right\rangle} B\left(\left\langle\mu_{t}\right\rangle\right)\right)^{2} \partial_{\left\langle\mu_{t}\right\rangle}\left(\frac{A\left(\left\langle\mu_{t}\right\rangle\right)}{B\left(\left\langle\mu_{t}\right\rangle\right)}\right)+B^{3}\left(\left\langle\mu_{t}\right\rangle\right) \partial_{\left\langle\mu_{t}\right\rangle}^{3}\left(\frac{A\left(\left\langle\mu_{t}\right\rangle\right)}{B\left(\left\langle\mu_{t}\right\rangle\right)}\right)\right)+\gamma_{1} B^{2}\left(\left\langle\mu_{t}\right\rangle\right) \\
& \times\left(1+\frac{2 \gamma_{2}}{\gamma_{1}} B\left(\left\langle\mu_{t}\right\rangle\right) \partial_{\left\langle\mu_{t}\right\rangle}\left(\frac{A\left(\left\langle\mu_{t}\right\rangle\right)}{B\left(\left\langle\mu_{t}\right\rangle\right)}\right)\right)+P_{t}\left(\gamma_{2} B^{3}\left(\left\langle\mu_{t}\right\rangle\right) \partial_{\left\langle\mu_{t}\right\rangle}^{3}\left(\frac{A\left(\left\langle\mu_{t}\right\rangle\right)}{B\left(\left\langle\mu_{t}\right\rangle\right)}\right)\right. \\
& +6 \gamma_{2} B^{2}\left(\left\langle\mu_{t}\right\rangle\right) \partial_{\left\langle\mu_{t}\right\rangle} B\left(\left\langle\mu_{t}\right\rangle\right) \partial_{\left\langle\mu_{t}\right\rangle}^{2}\left(\frac{A\left(\left\langle\mu_{t}\right\rangle\right)}{B\left(\left\langle\mu_{t}\right\rangle\right)}\right) \\
& +3 \gamma_{2} B^{2}\left(\left\langle\mu_{t}\right\rangle\right) \partial_{\left\langle\mu_{t}\right\rangle}^{2} B\left(\left\langle\mu_{t}\right\rangle\right) \partial_{\left\langle\mu_{t}\right\rangle}\left(\frac{A\left(\left\langle\mu_{t}\right\rangle\right)}{B\left(\left\langle\mu_{t}\right\rangle\right)}\right) \\
& +6 \gamma_{2} B\left(\left\langle\mu_{t}\right\rangle\right)\left(\partial_{\left\langle\mu_{t}\right\rangle} B\left(\left\langle\mu_{t}\right\rangle\right)\right)^{2} \partial_{\left\langle\mu_{t}\right\rangle}\left(\frac{A\left(\left\langle\mu_{t}\right\rangle\right)}{B\left(\left\langle\mu_{t}\right\rangle\right)}\right) \\
& \left.\left.+\gamma_{1} B\left(\left\langle\mu_{t}\right\rangle\right) \partial_{\left\langle\mu_{t}\right\rangle}^{2} B\left(\left\langle\mu_{t}\right\rangle\right)+\gamma_{1}\left(\partial_{\left\langle\mu_{t}\right\rangle} B\left(\left\langle\mu_{t}\right\rangle\right)\right)^{2}\right)\right) d t .
\end{aligned}
$$


Notably, the numerical coefficients of equations (13)-(14), approximate evolutions are attributed to the successive differentiation with respect to the term $\left\langle\mu_{t}\right\rangle$. Note that $\quad \partial_{\left\langle\mu_{t}\right\rangle}=\frac{\partial}{\partial\left\langle\mu_{t}\right\rangle}, \partial_{\left\langle\mu_{t}\right\rangle}^{2}=\frac{\partial^{2}}{\partial\left\langle\mu_{t}\right\rangle^{2}}, \partial_{\left\langle\mu_{t}\right\rangle}^{3}=\frac{\partial^{3}}{\partial\left\langle\mu_{t}\right\rangle^{3}} \quad$ and $\quad \partial_{\left\langle\mu_{t}\right\rangle}^{4}=\frac{\partial^{4}}{\partial\left\langle\mu_{t}\right\rangle^{4}} \quad$ are throughout the paper. For proof of equations (13)-(14), see the paper Patil and Sharma (2014).

Here, we state two special cases of evolution equations (13)-(14): (i) for the terms $\gamma_{1}=1$ and $\gamma_{2}=0$, equations (13)-(14) reduce to the classical exact evolution equations, see Eq. (4.159) of Jazwinski (1970, p. 137) (ii) the terms $\gamma_{1}=2 N$ and $\gamma_{2}=N \tau_{c o r}$ lead to the exact evolution equations of the OU process-driven SDE.

\section{POWER SYSTEM DYNAMICS AND NUMERICAL EXPERIMENTATIONS}

In deterministic setting, the swing equation of a single machine-infinite bus (SMIB) system is given by the following second-order non-linear differential equation Kundur (1994):

$$
M \ddot{\delta}+D \dot{\delta}+\frac{V E_{a}^{\prime}}{X} \sin \delta=P_{m}
$$

Note that the terms $V, M, D, E_{a}^{\prime}, \delta, X, P_{m}$ denotes the voltage magnitude of the infinite bus, combined inertia constant and the damping coefficient of the generator and turbine, the transient emf, the rotor angle of the generator, the total reactance, the input mechanical power respectively. After accounting random power fluctuations in the swing equation of the single machine-infinite bus system. we can get,

$$
M \ddot{\delta}+D \dot{\delta}+\frac{V E_{a}^{\prime}}{X} \sin \delta=P_{m}+\eta_{t},
$$

where $\eta_{t}$ is the OU process. After accomplishing the phase space formulation, we have $\mu_{1}=\delta_{t}, \mu_{2}=\dot{\delta}_{t}$,

$$
\dot{\mu}_{1}=\mu_{2}
$$




$$
\dot{\mu}_{2}=-\frac{D}{M} \mu_{2}-\frac{V E_{a}^{\prime}}{M X} \sin \mu_{1}+\frac{P_{m}}{M}+\frac{\eta_{t}}{M} .
$$

Consider the contribution to the evolution of the phase variable $\delta_{t}=\mu_{1}$ coming from the damping term is considerably greater than the inertial term (Hänggi and Jung, 1995, p. 241), then the term $-\frac{D}{M} \mu_{2}-\frac{V E_{a}^{\prime}}{M X} \sin \mu_{1}+\frac{P_{m}}{M}+\frac{\eta_{t}}{M}$ vanishes, i.e. If we model a system without physical or virtual inertia, power system is best described as a Kuramoto oscillator (Dörfler and Bullo 2010; Tönjes 2010; SimpsonPorco 2012; Schäfer et al. 2017; Supplemental material 2017) with the equation of motion,

$$
\dot{\delta}_{t}=-\frac{V E_{a}^{\prime}}{D X} \sin \delta_{t}+\frac{P_{m}}{D}+\frac{1}{D} \eta_{t}
$$

Thus, the above equation can be recast by utilizing a more convenient notation by choosing the state variable notation $\mu_{t}$ for the rotor angle $\delta_{t}$. Thus,

$$
\dot{\mu}_{t}=A\left(\mu_{t}, t\right)+B\left(\mu_{t}, t\right) \eta_{t},
$$

where

$$
A\left(\mu_{t}, t\right)=-\frac{V E_{a}^{\prime}}{D X} \sin \mu_{t}+\frac{P_{m}}{D}, B\left(\mu_{t}, t\right)=B(t)=\frac{1}{D}
$$

Note that for a non-linear time varying system. The input argument of the right-hand side of equation (20) involves the time variable $t$ as well. From equation (20) and equation (6) of the paper, we get

$$
d \mu_{t}=\left(-\frac{V E_{a}^{\prime}}{D X} \sin \mu_{t}\left(1+\frac{\gamma_{2}}{2 D^{2}}\right)+\frac{P_{m}}{D}\right) d t+\frac{\sqrt{\gamma_{1}}}{D} \sqrt{1-\frac{2 \gamma_{2}}{\gamma_{1}} \frac{V E_{a}^{\prime}}{D X} \cos \mu_{t} d} W_{t},
$$

After combining equations (13)-(14) with equation (20). As a result of these, we have the following system of prediction equations for the power system driven by the OU process,

$$
\begin{gathered}
d\left\langle\mu_{t}\right\rangle=\left(\frac{V E_{a}^{\prime}}{D X} \sin \left\langle\mu_{t}\right\rangle\left(1+\frac{N \tau_{c o r}}{2 D^{2}}\right)\left(\frac{P_{t}}{2}-1\right)+\frac{P_{m}}{D}\right) d t \\
d P_{t}=\left(P_{t} \frac{V E_{a}^{\prime}}{D X} \cos \left\langle\mu_{t}\right\rangle\left(-2+\frac{N \tau_{c o r}}{D^{2}}\right)-\frac{5}{2} N \tau_{c o r} \frac{V E_{a}^{\prime}}{D^{3} X} \cos \left\langle\mu_{t}\right\rangle+\frac{2 N}{D^{2}}\right) d t .
\end{gathered}
$$


Note that equation (20) and equation (21) are stochastically equivalent and suggest qualitative characteristics of the rotor angle of the machine. For numerical experimentations of equations (20)-(21), the initial conditions and system parameters for power system dynamics are the following Hirpara and Sharma (2015):

$$
\begin{gathered}
V=1.0 \mathrm{pu}, E_{a}^{\prime}=1.2 \mathrm{pu}, D=5 \mathrm{pu} / \mathrm{rad} / \mathrm{sec} \\
X_{d}^{\prime}=0.15 \mathrm{pu}, X_{l}=0.1 \mathrm{pu}, X=0.25 \mathrm{pu}, \tau_{c o r}=0.1 \\
P_{m}=1.0 \mathrm{pu},\langle\mu(0)\rangle=1 \mathrm{rad}, P(0)=0 \mathrm{rad}^{2}, N=0.05
\end{gathered}
$$

In first case, we consider that the correlation time $\tau_{c o r}$ is smaller than second case. Note that the solid line trajectory (-) of figure 1 demonstrates the numerical simulation of equation (20) and the dotted line (...) of figure 1 denotes the numerical simulation of equation (21) of the paper. The terms $\gamma_{1}=2 N$ and $\gamma_{2}=N \tau_{c o r}$ corresponding to correlation time $\tau_{c o r}=0.1$ become $\gamma_{1}=0.1$ and $\gamma_{2}=0.005$. Figure 1 suggested that qualitative characteristics of both SDEs conforms each other for a smaller correlation time. The trajectories of both SDEs are coinciding with each other. Thus, both SDEs are stochastically equivalent for the OU process with smaller correlation time. Figures 2 and 3 demonstrate numerical simulation of equation (22), mean trajectory and equation (23), variance trajectory with smaller correlation time.

For second case, we simulated equations (20) and (21) by choosing larger correlation time $\tau_{c o r}=1$ other parameters are same as first case. The terms $\gamma_{1}=0.1$ and $\gamma_{2}=0.05$ for larger correlation time. 


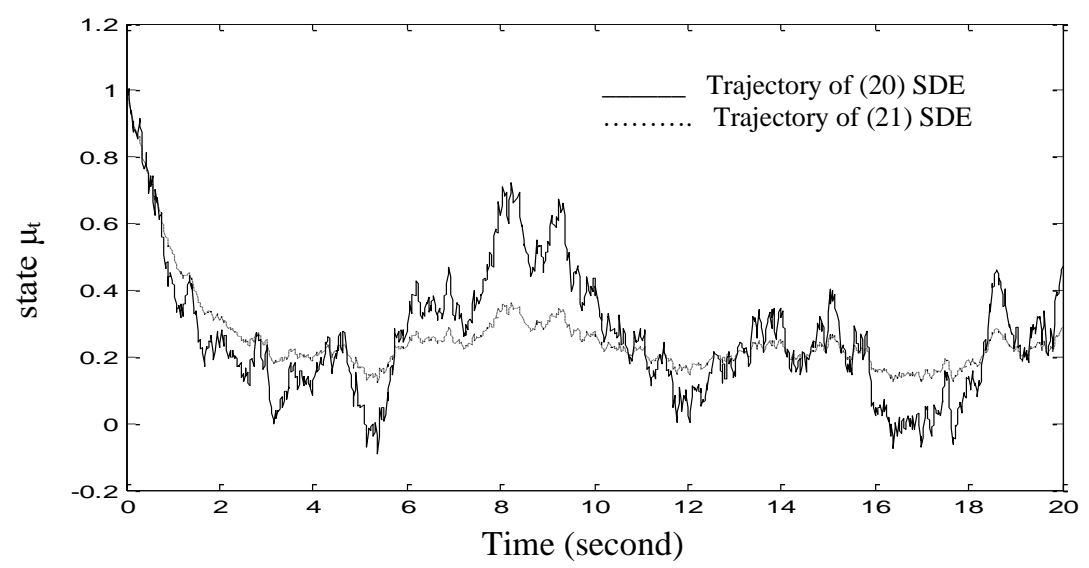

Figure 1: a comparison between trajectories

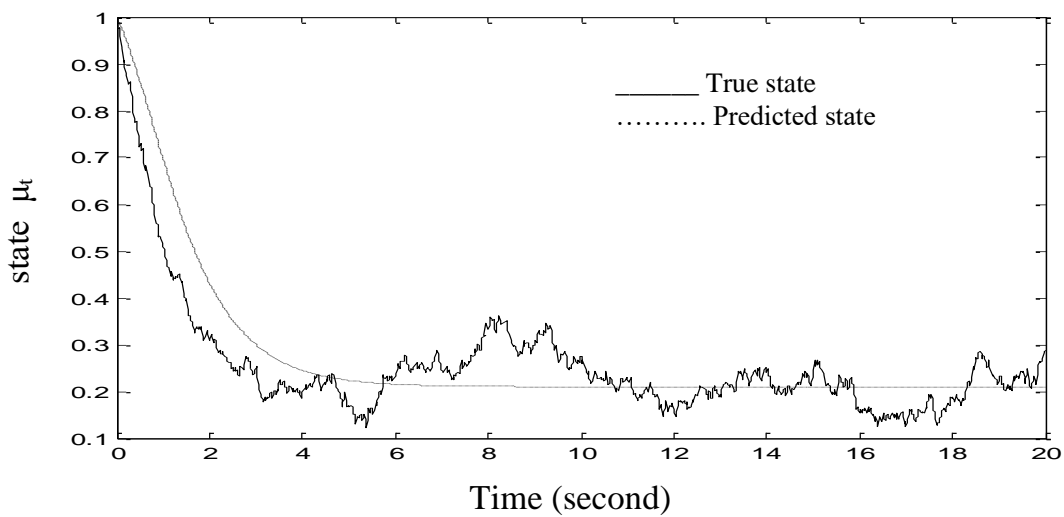

Figure 2: a comparison between trajectories

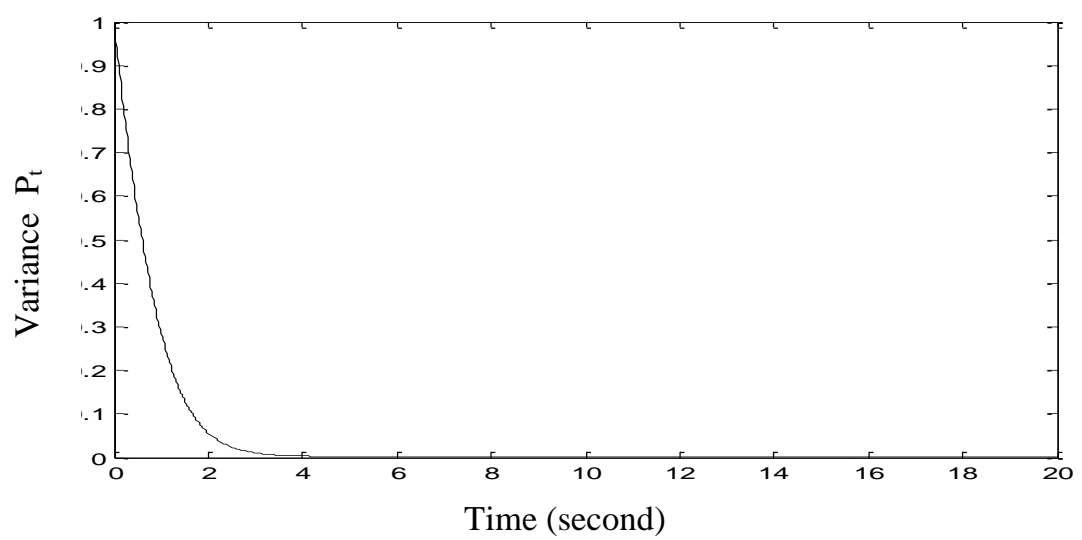

Figure 3: a variance trajectory 


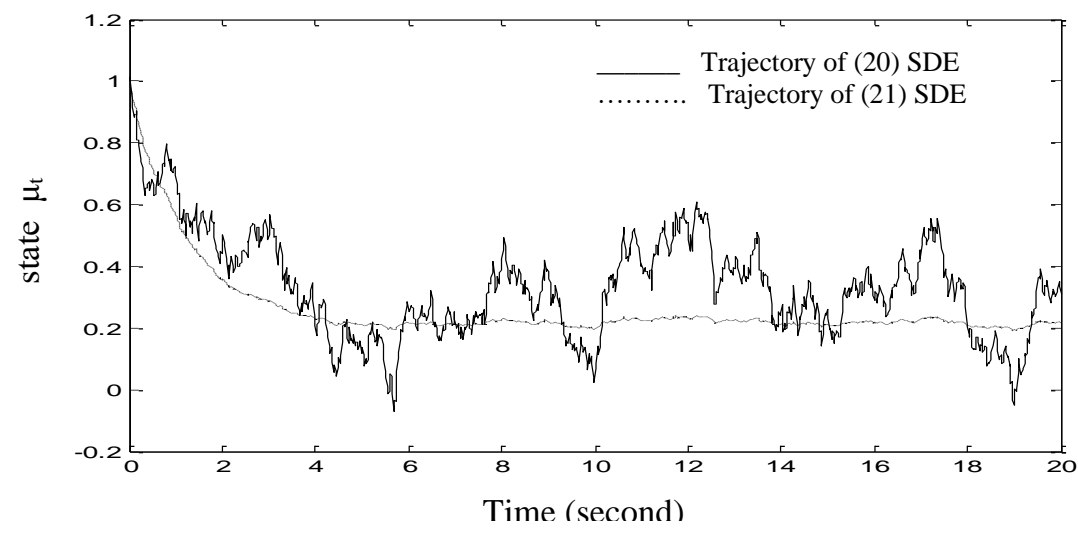

Figure 4: a comparison between trajectories

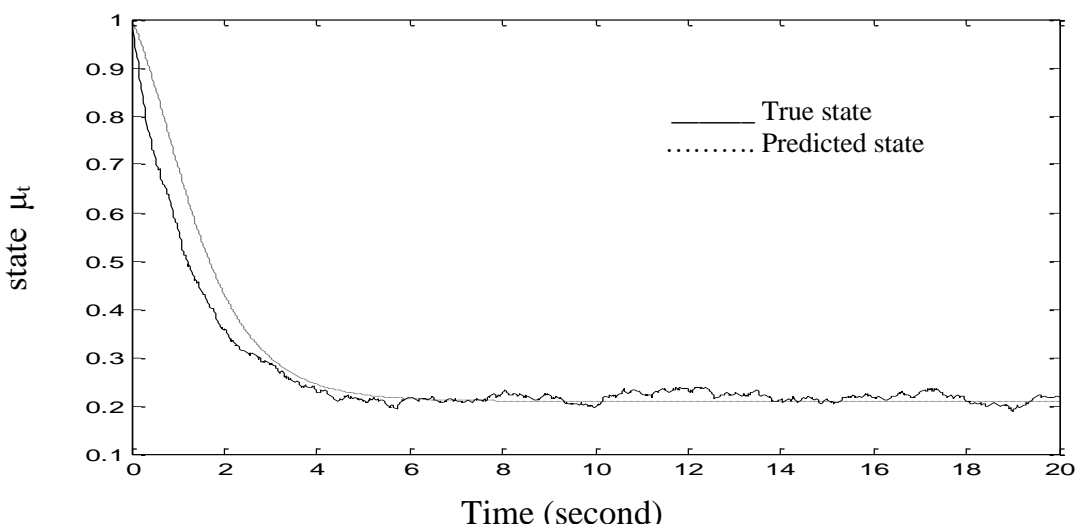

Figure 5: a comparison between trajectories

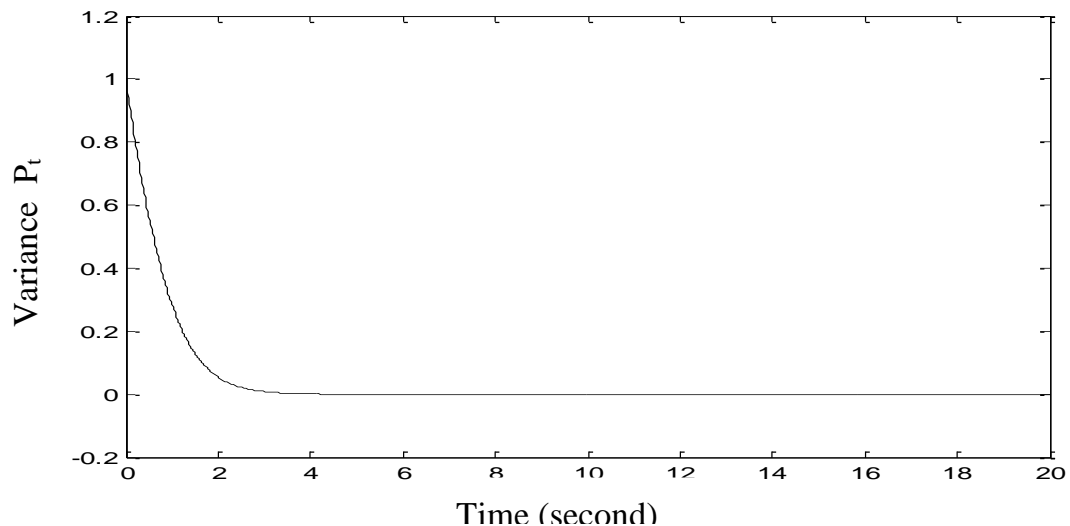

Figure 6: a variance trajectory 
The graphical notations are same as first case. Figure 4 reveals that the difference between the state trajectories resulting from the SDEs stated in equation (20) and equation (21) are larger. Figures 5 and 6 demonstrate numerical simulation of equation (22), mean trajectory and equation (23), variance trajectory with larger correlation time. Thus, the numerical simulation suggests that both SDEs, equation (20) and equation (21), are stochastically equivalent for the input coloured noise process with 'smaller correlation time'.

\section{CONCLUSION}

The main achievement of this paper is to develope prediction algorithm for the OU process-driven SDE. It is shown that for a smaller correlation time, the trajectories of SDEs stated in equation (20) and equation (21) are coinciding to each other. Thus, both SDEs are stochastically equivalent to each other and for larger correlation time, difference between both trajectories are larger. In this paper we have also developed conditional mean and variance equation for power system dynamics with stochastic equivalence approach. For future direction, in this paper we have considered no observation or value less observation. For available observation, we were required to develop filtering algorithm for the OU process driven power system dynamics.

\section{REFERENCES}

Dörfler F, Bullo F (2010) Synchronization and transient stability in power networks and non-uniform Kuramoto oscillators, Proceedings of the 2010 American Control Conference, 930-937 doi:10.1109/acc.2010.5530690

Guo SS, Shi Q (2017) Stationary solution of Duffing oscillator driven by additive and multiplicative Colored noise excitations, Journal of Vibration and Acoustics, 139(2): 024502-(1-4) doi:10.1115/1.4035308

Hänggi P, Jung P (1995) Colored noise in dynamical systems, Advances in Chemical Physics (I. Prigogine and S. A. Rice., eds.), John Wiley and Sons, New York, pp. 239-323

Hirpara RH (2019) On the stochasticity of power system dynamics using Stratonovich differential, IEEE INDICON-2019, 1-4 Doi: 10.1109/INDICON47234.2019.9030364

Hirpara RH, Sharma SN (2015) On the stochasticity of a machine swing equation using Itô differential, Proceedings of the ISCIE International Symposium on Stochastic Systems Theory and its Applications, 2015: 142-148 https://doi.org/10.5687/sss.2015.142 
Hirpara RH, Sharma SN (2015) An Ornstein-Uhlenbeck process-driven power system dynamics, IFAC PapersOnLine, 48(30):409-414 doi: 10.1016/j.ifacol.2015.12.413

Jazwinski AH (1970) Stochastic processes and filtering theory, Academic Press, New York and London

Kundur P (1994) Power system stability and control, Mc-Graw-Hill, New York

Patel HG, Sharma SN (2012) Some evolution equations for an Ornstein-Uhlenbeck process-driven dynamical system, Fluctuation and Noise Letters, 12(4): 1250020-(1-20) doi:10.1142/S0219477512500204

Patil NS, Sharma SN (2014) A prediction theory for a coloured noise-driven stochastic differential system, Systems Science \& Control Engineering, 2(1): 342-350 doi: $10.1080 / 21642583.2014 .906004$

Patil NS, Sharma SN (2015) On a non-linear stochastic dynamic circuit using Stratonovich differential, Journal of Franklin Institute, 352(8): 2999-3013 https://doi.org/10.1016/j.jfranklin.2014.12.018

Schäfer B, Matthiae M, Zhang X, Rohden M, Timme M, Witthaut D (2017) Escape Routes, Weak Links, and Desynchronization in Fluctuation-driven Networks, Physical Review E, 95: 060203-(1-5) doi: 10.1103/PhysRevE.95.060203

See Supplemental Material at http://link.aps.org/supplemental/10.1103/PhysRevE.95.060203 for discussion of robustness of the phenomena reported and a comparison of the escape time across different power grid models.

Simpson-Porco JW, Dörfler F, Bullo F (2012) Droop-controlled inverters are Kuramoto oscillators, IFAC Proceedings Volumes, 45(26): 264-269 doi:10.3182/20120914-2-US-4030.00055

Stratonovich RL (1963) Topics in the theory of random noise, Vol. 1, Gordon and Breach, New York

Tönjes R (2010) Synchronization transition in the Kuramoto model with colored noise, Physical Review E, 81: 055201-(1-4) doi: 10.1103/PhysRevE.81.055201

Verdejo H, Awerkin A, Kliemann W, Becker C (2019) Modelling uncertanities in electrical power systems with stochastic differential equations, International Journal of Electrical Power \& Energy Systems, 113:322-332 doi: 10.1016/j.ijepes.2019.05.054

Wang K, Crow ML (2013) The Fokker-Planck equation for power system stability probability density function evolution, IEEE Transactions on Power Systems, 28(3): 2994-3001 Doi: 10.1109/TPWRS.2012.2232317

Ravish H. Hirpara, Ph.D., Associate Professor, Electrical Engineering Department, S.N. Patel Institute of Technology and Research Centre,Umrakh, Bardoli, Surat-394345, Gujarat, India

E-Mail: ravishhirpara@gmail.com, ravish.hirpara@snpitrc.ac.in M: +919924259911

Received April 2020 